\title{
A SYSTEM OF COUPLED SCHRÖDINGER EQUATIONS WITH TIME-OSCILLATING NONLINEARITY
}

\author{
X. CARVAJAL, P. GAMBOA AND M. PANTHEE
}

ABstract. This paper is concerned with the initial value problem (IVP) associated to the coupled system of supercritical nonlinear Schrödinger equations

$$
\left\{\begin{array}{l}
i u_{t}+\Delta u+\theta_{1}(\omega t)\left(|u|^{2 p}+\beta|u|^{p-1}|v|^{p+1}\right) u=0 \\
i v_{t}+\Delta v+\theta_{2}(\omega t)\left(|v|^{2 p}+\beta|v|^{p-1}|u|^{p+1}\right) v=0
\end{array}\right.
$$

where $\theta_{1}$ and $\theta_{2}$ are periodic functions, which has applications in many physical problems, especially in nonlinear optics. We prove that, for given initial data $\varphi, \psi \in$ $H^{1}\left(\mathbb{R}^{n}\right)$, as $|\omega| \rightarrow \infty$, the solution $\left(u_{\omega}, v_{\omega}\right)$ of the IVP $(0.1)$ converges to the solution $(U, V)$ of the IVP associated to

$$
\left\{\begin{array}{l}
i U_{t}+\Delta U+I\left(\theta_{1}\right)\left(|U|^{2 p}+\beta|U|^{p-1}|V|^{p+1}\right) U=0 \\
i V_{t}+\Delta V+I\left(\theta_{2}\right)\left(|V|^{2 p}+\beta|V|^{p-1}|U|^{p+1}\right) V=0
\end{array}\right.
$$

with the same initial data, where $I(g)$ is the average of the periodic function $g$. Moreover, if the solution $(U, V)$ is global and bounded, then we prove that the solution $\left(u_{\omega}, v_{\omega}\right)$ is also global provided $|\omega| \gg 1$.

\section{INTRODUCTION}

In this work, we consider the following initial value problem (IVP) for two coupled nonlinear Schrödinger (NLS) equations:

$$
\left\{\begin{array}{l}
i u_{t}+\Delta u+\theta_{1}(\omega t)\left(|u|^{2 p}+\beta|u|^{p-1}|v|^{p+1}\right) u=0 \\
i v_{t}+\Delta v+\theta_{2}(\omega t)\left(|v|^{2 p}+\beta|v|^{p-1}|u|^{p+1}\right) v=0 \\
u\left(x, t_{0}\right)=\varphi(x), \quad v\left(x, t_{0}\right)=\psi(x),
\end{array}\right.
$$

2000 Mathematics Subject Classification. 35A07, 35Q53.

Key words and phrases. Schrödinger equation, Initial value problem, Strichartz estimate, Wellposedness.

X.C. was partially supported by Pronex-FAPERJ, Brazil, through the grant E-26/110.560/2010.

M. P. was partially supported by FEDER Funds through "COMPETE"; Portuguese Funds through FCT - within the Project Est-C/MAT/UI0013/2011. 
in $\mathbb{R}^{n}$, where

$$
0<p<\frac{2}{(n-2)^{+}}
$$

$t_{0}, \omega \in \mathbb{R}$ and $u, v: \mathbb{R}^{n} \times \mathbb{R} \rightarrow \mathbb{C}$, and $\theta_{1}, \theta_{2} \in C(\mathbb{R}, \mathbb{R})$ are periodic functions with period $\tau>0$. Moreover, $\beta$ is real positive constant. To simplify the analysis, we translate the initial time $t_{0}$ to 0 and consider the following IVP

$$
\left\{\begin{array}{l}
i u_{t}+\Delta u+\theta_{1}\left(\omega\left(t+t_{0}\right)\right)\left(|u|^{2 p}+\beta|u|^{p-1}|v|^{p+1}\right) u=0 \\
i v_{t}+\Delta v+\theta_{2}\left(\omega\left(t+t_{0}\right)\right)\left(|v|^{2 p}+\beta|v|^{p-1}|u|^{p+1}\right) v=0 \\
u(x, 0)=\varphi(x), \quad v(x, 0)=\psi(x) .
\end{array}\right.
$$

For $\theta_{1}=\theta_{2}=1$ this kind of problem arises as a model for propagation of polarized laser beams in birefringent Kerr medium in nonlinear optics (see, for example, $[2,9,11,14,19,20]$ and the references therein for a complete discussion of the physics of the problem). The two functions $u$ and $v$ are the components of the slowly varying envelope of the electrical field, $t$ is the distance in the direction of propagation, $x$ are orthogonal variables and $\Delta$ is the diffraction operator. The case $n=1$ corresponds to propagation in a planar geometry, $n=2$ is the propagation in a bulk medium and $n=3$ is the propagation of pulses in a bulk medium with time dispersion. The focusing nonlinear terms in (1.1) describes the dependence of the refraction index of material on the electric field intensity and the birefringence effects. The parameter $\beta>0$ has to be interpreted as the birefringence intensity and describes the coupling between the two components of the electric-field envelope.

This article is motivated by the works of Abdullaev et al. [1] and Konotop and Pacciani [10], where the authors investigate the effect of a time-oscillating term in factor of nonlinearity of the nonlinear Schrödinger equation and Carvajal, Panthee and Scialom [4], where the authors considered a critical Korteweg-de Vries (KdV) equation with time-oscillating nonlinearity.

If we consider the system (1.1) for $\theta_{1}=\theta_{2}=1, t_{0}=0$ i.e.

$$
\left\{\begin{array}{l}
i u_{t}+\Delta u+\left(|u|^{2 p}+\beta|u|^{p-1}|v|^{p+1}\right) u=0 \\
i v_{t}+\Delta v+\left(|v|^{2 p}+\beta|v|^{p-1}|u|^{p+1}\right) v=0 \\
u(x, 0)=\varphi(x), \quad v(x, 0)=\psi(x)
\end{array}\right.
$$


then the system (1.4), admits the mass and the energy conservation in the spaces $L^{2}\left(\mathbb{R}^{n}\right) \times L^{2}\left(\mathbb{R}^{n}\right)$ and $H^{1}\left(\mathbb{R}^{n}\right) \times H^{1}\left(\mathbb{R}^{n}\right)$ respectively. More precisely, the mass $\left(L^{2}\right.$ norm):

$$
M[u(t), v(t)]:=\|\varphi\|_{L^{2}\left(\mathbb{R}^{n}\right)}^{2}+\|\psi\|_{L^{2}\left(\mathbb{R}^{n}\right)}^{2},
$$

and the energy:

$$
\begin{aligned}
E[u(t), v(t)]:= & \frac{1}{2}\left(\|\nabla u(t)\|_{L^{2}\left(\mathbb{R}^{n}\right)}^{2}+\|\nabla v(t)\|_{L^{2}\left(\mathbb{R}^{n}\right)}^{2}\right) \\
& -\frac{1}{2 p+2}\left[\|u(t)\|_{L^{2 p+2}\left(\mathbb{R}^{n}\right)}^{2 p+2}+2 \beta\|u(t) v(t)\|_{L^{p+1}\left(\mathbb{R}^{n}\right)}^{p+1}+\|v(t)\|_{L^{2 p+2}\left(\mathbb{R}^{n}\right)}^{2 p+2}\right] \\
= & E[\varphi, \psi],
\end{aligned}
$$

are conserved by the flow of (1.4). For some remarks on proofs of conservation laws for nonlinear Schrödinger equations, we refer to [15].

Well-posedness issues and the blow-up phenomenon for the IVP (1.4) has been extensively studied in the literature, see for example in $[6,8,9,13,14,16,19]$ and references therein.

In what follows we list some important results that are relevant in our work.

1) When $1<p<2 / n$, the solution of the Cauchy problem (1.4), exists globally in time (see [9]).

2) When $p \geq 2 / n$, the solution of the Cauchy problem (1.4), blows-up in a finite time for some initial data (satisfying $E[\varphi, \psi]<0$ ), especially for a class of sufficiently large data (see $[8,9,13,16])$. On the other hand, the solution of the Cauchy problem (1.4) exists globally for other initial data, especially for a class of sufficiently small data (see $[6,9,14])$.

In the sections 2,3 and 4 we will study the Cauchy problem and the blow-up phenomenon for the system $(1.1)$ with $\theta_{1}, \theta_{2} \in L^{\infty}(\mathbb{R})$.

In [19], Xiaoguang et al. obtained a sharp threshold of blow-up solution for (1.4). To study the blow-up threshold, the following stationary system

$$
\left\{\begin{array}{l}
\Delta Q-\frac{(2-n) p+2}{2} Q+\left(|Q|^{2 p}+\beta|Q|^{p-1}|R|^{p+1}\right) Q=0, \\
\Delta R-\frac{(2-n) p+2}{2} R+\left(|R|^{2 p}+\beta|R|^{p-1}|Q|^{p+1}\right) R=0
\end{array}\right.
$$

associated with (1.4) was considered. 


$$
\begin{gathered}
\text { For, } s_{c}=n / 2-1 / p \text {, defining } \sigma_{p, n, \beta}:=\left(\frac{p n}{2}\right)^{2 p} \sqrt{\|Q\|_{L^{2}\left(\mathbb{R}^{n}\right)}^{2}+\|R\|_{L^{2}\left(\mathbb{R}^{n}\right)}^{2}}, \\
\Gamma[u, v]:=E^{s_{c}}[u, v] M^{1-s_{c}}[u, v]
\end{gathered}
$$

and

$$
\vartheta[u, v]:=\left(\|\nabla u\|_{L^{2}\left(\mathbb{R}^{n}\right)}^{2}+\|\nabla v\|_{L^{2}\left(\mathbb{R}^{n}\right)}^{2}\right)^{s_{c} / 2}\left(\|u\|_{L^{2}\left(\mathbb{R}^{n}\right)}^{2}+\|v\|_{L^{2}\left(\mathbb{R}^{n}\right)}^{2}\right)^{\left(1-s_{c}\right) / 2},
$$

the following is the result proved in Xiaoguang et al. [19].

Theorem 1.1 ([19]). Let $\frac{2}{n} \leq p<A_{n}$, where $A_{n}=\infty$ if $n=1,2, A_{n}=2 /(n-2)$ if $n \geq 3$ and $(|x| \varphi,|x| \psi) \in L^{2}\left(\mathbb{R}^{n}\right) \times L^{2}\left(\mathbb{R}^{n}\right)$. Assume that

$$
\Gamma[\varphi, \psi]<\Gamma[Q, R] \equiv\left(\frac{s_{c}}{n}\right)^{s_{c}}\left(\sigma_{p, n, \beta}\right)^{2},
$$

then the following two conclusions are valid.

1) If $\vartheta[\varphi, \psi]<\vartheta[Q, R]$, then the solution exists globally in time.

2) If $\vartheta[\varphi, \psi]>\vartheta[Q, R]$, then the solution blows-up in finite time.

Now, coming back to our problem, note that the system (1.3) is equivalent to the following integral equation form

$$
\left\{\begin{array}{l}
u(t)=S(t) \varphi+i \int_{0}^{t} S(t-s) \theta_{1}\left(\omega\left(s+t_{0}\right)\right) F(u, v)(s) d s \\
v(t)=S(t) \psi+i \int_{0}^{t} S(t-s) \theta_{2}\left(\omega\left(s+t_{0}\right)\right) F(v, u)(s) d s
\end{array}\right.
$$

where $S(t)=e^{i t \Delta}$ is the group that describes the linear flow of the Schrödinger equation and $F(u, v):=\left(|u|^{2 p}+\beta|u|^{p-1}|v|^{p+1}\right) u$. Using standard method we can see that the system (1.8) is locally well-posed for given data in $H^{1}\left(\mathbb{R}^{n}\right) \times H^{1}\left(\mathbb{R}^{n}\right)$. In fact, we have the following result from [7].

Proposition $1.1([7])$. Let $p$ be as in (1.2). Given any $(\varphi, \psi) \in H^{1}\left(\mathbb{R}^{n}\right) \times H^{1}\left(\mathbb{R}^{n}\right)$, $\theta_{1}, \theta_{2} \in L^{\infty}(\mathbb{R})$ and $t_{0} \in \mathbb{R}$, there exists a unique, maximal solution

$$
(u, v) \in \mathbb{C}\left(\left[0, T_{\max }\right), H^{1}\right),
$$

of (1.8). Also the solution satisfies the blow-up alternative, i.e. if $T_{\max }<\infty$ then $\|\mid(u(t), v(t))\|_{1,2} \rightarrow \infty$ as $t \rightarrow T_{\max }$. Moreover

$$
(u, v) \in \mathbb{L}^{q}\left((0, T), W^{1, r}\right) \quad \text { for } \quad 0<T<T_{\max },
$$

for all admissible pairs $(q, r)$. 
As stated earlier, the purpose of this paper is to study the behavior of the solution $\left(u_{t_{0}, \omega}, v_{t_{0}, \omega}\right)$ of the IVP (1.3) (or equivalently of (1.8)) as $|\omega| \rightarrow \infty$. It is natural to expect that the nonlinearity averages to $\left\{I\left(\theta_{1}\right)\left(|U|^{2 p}+\beta|U|^{p-1}|V|^{p+1}\right) U, I(\theta)\left(|V|^{2 p}+\right.\right.$ $\left.\left.\beta|V|^{p-1}|U|^{p+1}\right) V\right\}$ as $|\omega| \rightarrow \infty$, where $I(h)$ is the average of a $\tau$-periodic function $h$, i.e.,

$$
I(h):=\frac{1}{\tau} \int_{0}^{\tau} h(s) d s .
$$

So, one may expect that the solution $\left(u_{t_{0}, \omega}, v_{t_{0}, \omega}\right)$ of the system (1.8) converges locally in time, as $|\omega| \rightarrow \infty$, to solution $(U, V)$ of

$$
\left\{\begin{array}{l}
i U_{t}+\Delta U+I\left(\theta_{1}\right)\left(|U|^{2 p}+\beta|U|^{p-1}|V|^{p+1}\right) U=0 \\
i V_{t}+\Delta V+I\left(\theta_{2}\right)\left(|V|^{2 p}+\beta|V|^{p-1}|U|^{p+1}\right) V=0 \\
U(x, 0)=\varphi(x), \quad V(x, 0)=\psi(x),
\end{array}\right.
$$

or equivalently

$$
\left\{\begin{array}{l}
U(t)=S(t) \varphi+i I\left(\theta_{1}\right) \int_{0}^{t} S(t-s) F(U, V)(s) d s \\
V(t)=S(t) \psi+i I\left(\theta_{2}\right) \int_{0}^{t} S(t-s) F(V, U)(s) d s .
\end{array}\right.
$$

This is indeed what the first main result of this work shows in following theorem.

Theorem 1.2. Assume (1.2). Fix an initial value $(\varphi, \psi) \in H^{1}\left(\mathbb{R}^{n}\right) \times H^{1}\left(\mathbb{R}^{n}\right)$. Given $t_{0}, \omega \in \mathbb{R}$, denote by $\left(u_{t_{0}, \omega}, v_{t_{0}, \omega}\right)$ the maximal solution of (1.8). Let $(U, V)$ be the solution of (1.11) defined on the maximal interval $\left[0, S_{\max }\right)$.

- Given any $0<T<S_{\text {max }}$, the solution $\left(u_{t_{0}, \omega}, v_{t_{0}, \omega}\right)$ exists on $[0, T]$ for all $t_{0} \in \mathbb{R}$ provided $|\omega|$ is sufficient large.

- We have that $\left(u_{t_{0}, \omega}, v_{t_{0}, \omega}\right) \rightarrow(U, V)$ in $\mathbb{L}^{\gamma}\left((0, T), W^{1, \rho}\right)$ as $|\omega| \rightarrow \infty$, uniformly in $t_{0} \in \mathbb{R}$, for all admissible pairs $(\gamma, \rho)$ and all $0<T<S_{\max }$. In particular, convergence holds in $\mathbb{C}\left([0, T], H^{1}\right)$ for all $0<T<S_{\text {max }}$.

Whenever $S_{\max }=\infty$, one may wonder whether or not $\left(u_{t_{0}, \omega}, v_{t_{0}, \omega}\right)$ is global when $|\omega|$ is sufficiently large. The following theorem, the second main result of this work, shows that the answer is positive provided $(U, V)$ has sufficient decay as $t \rightarrow \infty$.

Theorem 1.3. Assume (1.2). Set

$$
r=2(\alpha+1) \quad a=\frac{4 p(p+1)}{2-p(n-2)} .
$$


Fix the initial data $(\varphi, \psi) \in H^{1}\left(\mathbb{R}^{n}\right) \times H^{1}\left(\mathbb{R}^{n}\right)$. For $t_{0}, \omega \in \mathbb{R}$ denote by $\left(u_{t_{0}, \omega}, v_{t_{0}, \omega}\right)$ the maximal solutions of (1.8). Suppose $(U, V)$ be the maximal solution of (1.11) defined in the maximal interval $\left[0, S_{\max }\right)$. If $S_{\max }=\infty$ and

$$
(U, V) \in \mathbb{L}^{a}\left((0, \infty), L^{r}\left(\mathbb{R}^{n}\right)\right),
$$

then it follows that $\left(u_{t_{0}, \omega}, v_{t_{0}, \omega}\right)$ is global for all $t_{0} \in \mathbb{R}$ if $|\omega|$ is sufficiently large. Moreover, $\left(u_{t_{0}, \omega}, v_{t_{0}, \omega}\right) \rightarrow(U, V)$ in $\mathbb{L}^{\gamma}\left((0, \infty), W^{1, \rho}\right)$ as $|\omega| \rightarrow \infty, t_{0} \in \mathbb{R}$ for all admissible pairs $(\gamma, \rho)$. In particular, the convergence holds in $\mathbb{L}^{\infty}\left((0, \infty), H^{1}\right)$.

The rest of the paper is organized as follows. In Section 2, we obtain some preliminary results. In Section 3, we prove Lemma 3.1 and in the Section 4, we prove the main results of this work, Theorems 1.2 and 1.3.

\section{NOTATION}

The $L^{2}$-based Sobolev space of order s will be denoted by $H^{s}$ with norm

$$
\|f\|_{H^{s}}:=\left(\int_{\mathbb{R}^{n}}\left(1+|\xi|^{2}\right)^{s}|\hat{f}(\xi)|^{2} d \xi\right)^{1 / 2} .
$$

For $f:[0, T] \times \mathbb{R}^{n} \rightarrow \mathbb{R}$ we define the mixed $L_{t}^{p} L_{x}^{q}$-norm by

$$
\|f\|_{L_{T}^{p} L_{x}^{q}}:=\left\{\int_{0}^{T}\left[\int_{\mathbb{R}^{n}}|f(x, t)|^{q} d t\right]^{p / q} d x\right\}^{1 / p},
$$

with usual modifications when $p=\infty$. We use the letter $C$ to denote various constants whose exact values are immaterial and which may vary from one line to the next. Also, we use the following notations throughout the text.

$$
\begin{aligned}
& \mathbb{L}^{r}\left(\mathbb{R}^{n}\right)=L^{r}\left(\mathbb{R}^{n}\right) \times L^{r}\left(\mathbb{R}^{n}\right) . \\
& \mathbb{H}^{s}\left(\mathbb{R}^{n}\right)=H^{s}\left(\mathbb{R}^{n}\right) \times H^{s}\left(\mathbb{R}^{n}\right) . \\
& \|\cdot\|_{\infty}=\|\cdot\|_{L^{\infty}} . \\
& \|\cdot\|_{1,2}=\|\cdot\|_{H^{1}\left(\mathbb{R}^{n}\right)} \cdot \\
& \||(\cdot, \cdot)|\|_{1,2}=\|\cdot\|_{1,2}+\|\cdot\|_{1,2} . \\
& L_{\infty}^{a} L_{x}^{r}:=L^{a}\left((0, \infty), L^{r}\left(\mathbb{R}^{n}\right)\right) . \\
& L_{T}^{a} L_{x}^{r}:=L^{a}\left((0, T), L^{r}\left(\mathbb{R}^{n}\right)\right) \\
& \mathbb{L}^{a}\left((0, T), L^{r}\right):=L_{T}^{a} L_{x}^{r} \times L_{T}^{a} L_{x}^{r} . \\
& F(u, v):=\left(|u|^{2 p}+\beta|u|^{p-1}|v|^{p+1}\right) u . \\
& \mathbb{C}\left([a, b], H^{1}\right):=C\left([a, b], H^{1}\left(\mathbb{R}^{n}\right)\right) \times C\left([a, b], H^{1}\left(\mathbb{R}^{n}\right)\right) . \\
& \mathbb{L}^{p}\left((a, b), W^{1, q}\right):=L^{p}\left((a, b), W^{1, q}\left(\mathbb{R}^{n}\right)\right) \times L^{p}\left((a, b), W^{1, q}\left(\mathbb{R}^{n}\right)\right) .
\end{aligned}
$$




\section{Preliminary RESUlts}

Given $1 \leq p \leq \infty$, we denote by $p^{\prime}$ its conjugate given by $\frac{1}{p^{\prime}}:=1-\frac{1}{p}$. We use the standard Sobolev spaces and their embedding. We consider the standard notion of a (non-endpoint) admissible pair $(q, r)$, i.e.

$$
\frac{2}{q}:=\frac{n}{2}-\frac{n}{r}, \quad 2 \leq r<\frac{2 n}{(n-2)^{+}} .
$$

We will use the Strichartz estimates satisfied by the linear Schrödinger group, more precisely, given any two admissible pairs $(q, r)$ and $(\gamma, \rho)$, there exists a constant $\mathrm{C}$ such that if

$$
u(t)=S(t) \varphi+i \int_{0}^{t} S(t-s) f(s) d s
$$

then

$$
\|u\|_{\mathbb{L}^{q}\left(\mathbb{R}, L^{r}\left(\mathbb{R}^{n}\right)\right)} \leq C\left(\|\varphi\|_{L^{2}\left(\mathbb{R}^{n}\right)}+\|f\|_{L^{\gamma^{\prime}}\left(\mathbb{R}, L^{\left.\rho^{\prime}\left(\mathbb{R}^{n}\right)\right)}\right)} .\right.
$$

In this section, we first recall some results concerning the local and global wellposedness for (1.8). Next, we study the effect of the oscillating term $\theta(\omega t)$ as $|\omega| \rightarrow \infty$ on the linear, nonhomogeneus Schrödinger equation. Given $h, z \in L^{\infty}(\mathbb{R})$, we consider the equation

$$
\left\{\begin{array}{l}
u(t)=S(t) \varphi+i \int_{0}^{t} S(t-s) h(s) F(u, v)(s) d s, \\
v(t)=S(t) \psi+i \int_{0}^{t} S(t-s) z(s) F(v, u)(s) d s,
\end{array}\right.
$$

which is slightly more general than (1.3).

Proposition 2.1. (Local Existence) Assume (1.2). Given $A, M>0$, such that

$$
\begin{array}{rll}
\|h\|_{\infty}+\|z\|_{\infty} \leq A & \text { for all } & h, z \in L^{\infty}\left(\mathbb{R}^{n}\right), \\
\|\varphi\|_{1,2}+\|\psi\|_{1,2} \leq M & \text { for all } & \varphi, \psi \in H^{1}\left(\mathbb{R}^{n}\right),
\end{array}
$$

then there exist $\delta=\delta(A, M)$ and a unique solution $(u, v) \in \mathbb{C}\left([0, \delta], H^{1}\right)$ of (2.3). In addition

$$
\|(u, v)\|_{\mathbb{L}^{\infty}\left((0, \delta), H^{1}\right)} \leq C\left\{\|\varphi\|_{1,2}+\|\psi\|_{1,2}\right\} .
$$

Moreover, $(u, v) \in \mathbb{L}^{\gamma}\left((0, \delta), W^{1, \rho}\right)$ for all admissible pairs $(\gamma, \rho)$.

Proof. For the proof of this result we refer to [7] or [17].

We will also use the following result. 
Proposition 2.2. Assume (1.2). Let $r, q, a$ be defined by

$$
r:=2(p+1), \quad q:=\frac{4(p+1)}{p n}, \quad a:=\frac{4 p(p+1)}{2-p(n-2)},
$$

such that $a>\frac{q}{2}$. Given any $A>0$, there exists $\varepsilon=\varepsilon(A)$ and $\Lambda$ such that if

$$
\begin{aligned}
\|h\|_{\infty}+\|z\|_{\infty} & \leq A, \\
\|S(\cdot)(\varphi, \psi)\|_{\mathbb{L}^{a}\left((0, \infty), L^{r}\right)} & \leq \varepsilon, \quad \text { for all } \varphi, \psi \in H^{1}\left(\mathbb{R}^{n}\right),
\end{aligned}
$$

then the corresponding solution $(u, v)$ of (2.3) is global and satisfies

$$
\|(u, v)\|_{\mathbb{L}^{a}\left((0, \infty), L^{r}\right)} \leq C\|S(\cdot)(\varphi, \psi)\|_{\mathbb{L}^{a}\left((0, \infty), L^{r}\right)}
$$

and

$$
\|(u, v)\|_{\mathbb{L}^{q}\left((0, \infty), W^{1, r}\right)}+\|(u, v)\|_{\mathbb{L}^{\infty}\left((0, \infty), H^{1}\right)} \leq \Lambda \mid\|(\varphi, \psi)\| \|_{1,2} .
$$

Conversely, if the solution $(u, v)$ of (2.3) is global and satisfies

$$
\|(u, v)\|_{\mathbb{L}^{a}\left((0, \infty), L^{r}\right)} \leq \epsilon,
$$

then

$$
\|S(\cdot)(\varphi, \psi)\|_{\mathbb{L}^{a}\left((0, \infty), L^{r}\right)} \leq c\|(u, v)\|_{\mathbb{L}^{a}\left((0, \infty), L^{r}\right)} .
$$

Proof. Let $G_{h, u, v}(t):=i \int_{0}^{t} S(t-s) h(s) F(u, v)(s) d s$. From (2.3), we obtain that

$$
\left\{\begin{array}{l}
u(t)=S(t)+G_{h, u, v}(t), \\
v(t)=S(t)+G_{z, v, u}(t) .
\end{array}\right.
$$

Then

$$
\left|\|u\|_{L_{T}^{a} L_{x}^{r}}-\left\|\left.S(\cdot) \varphi\right|_{L_{T}^{a} L_{x}^{r}} \mid \leq\right\| G_{h, u, v} \|_{L_{T}^{a} L_{x}^{r}}, \quad \forall 0<T<T_{\max }\right.
$$

and

$$
\left|\|v\|_{L_{T}^{a} L_{x}^{r}}-\|S(.) \psi\|_{L_{T}^{a} L_{x}^{r}}\right| \leq\left\|G_{z, v, u}\right\|_{L_{T}^{a} L_{x}^{r}}, \quad \forall 0<T<T_{\max } .
$$

From (2.2), (2.3), (2.15) and (2.16), we obtain

$$
\|u\|_{L_{T}^{a} L_{x}^{r}} \leq\|S(\cdot) \varphi\|_{L_{T}^{a} L_{x}^{r}}+C A\left\{\|u\|_{L_{T}^{a} L_{x}^{r}}^{2 p+1}+\beta\|v\|_{L_{T}^{a} L_{x}^{r}}^{p+1}\|u\|_{L_{T}^{a} L_{x}^{r}}^{p}\right\}
$$

and

$$
\|v\|_{L_{T}^{a} L_{x}^{r}} \leq\|S(\cdot) \psi\|_{L_{T}^{a} L_{x}^{r}}+C A\left\{\|v\|_{L_{T}^{a} L_{x}^{r}}^{2 p+1}+\beta\|u\|_{L_{T}^{a} L_{x}^{r}}^{p+1}\|v\|_{L_{T}^{a} L_{x}^{r}}^{p}\right\} .
$$

The estimates (2.17) and (2.18), yield

$$
\|(u, v)\|_{\mathbb{L}^{a}\left((0, T), L^{r}\right)} \leq\|S(\cdot)\{\varphi, \psi\}\|_{\mathbb{L}^{a}\left((0, T), L^{r}\right)}+C A\left\{X_{T}(u, v)+X_{T}(v, u)\right\},
$$


where

$$
X_{T}(u, v):=\|u\|_{L_{T}^{a} L_{x}^{r}}^{2 p+1}+\beta\|v\|_{L_{T}^{a} L_{x}^{r}}^{p+1}\|u\|_{L_{T}^{a} L_{x}^{r}}^{p} .
$$

Let $\epsilon=\epsilon(A)$ be small enough so that

$$
(1+2 \beta) 2^{2 p+2} \epsilon^{2 p} C A<1 .
$$

From (2.9) and (2.19), we can say that

$$
\|(u, v)\|_{\mathbb{L}^{a}\left((0, T), L^{r}\right)} \leq \epsilon+C A\left\{X_{T}(u, v)+X_{T}(v, u)\right\}, \quad \forall 0<T<T_{\max } .
$$

We will show that

$$
\|(u, v)\|_{\mathbb{L}^{a}\left((0, T), L^{r}\right)} \leq 2 \epsilon \quad \forall 0 \leq T \leq T_{\max } .
$$

We use contradiction method to prove (2.22). Suppose that there is $T_{*} \in\left[0, T_{\max }\right]$ such that

$$
f\left(T_{*}\right)>2 \epsilon \quad \text { where } \quad f(T):=\|(u, v)\|_{\mathbb{L}^{a}\left((0, T), L^{r}\right)} \quad 0<T<T_{\max } .
$$

As $f(t)$ is a continuous function and increasing in $0<T<T_{\max }$, there is $0<T_{0}<T_{*}$ such that

$$
f\left(T_{0}\right):=\|(u, v)\|_{\mathbb{L}^{a}\left(\left(0, T_{0}\right), L^{r}\right)}=2 \epsilon .
$$

We observe that, from $(2.21)$

$$
\begin{aligned}
f\left(T_{0}\right) & \leq \epsilon+C A\left\{X_{T_{0}}(u, v)+X_{T_{0}}(v, u)\right\}, \\
& \leq \epsilon+C A\left\{(2 \epsilon)^{2 p+1}+\beta(2 \epsilon)^{2 p+1}+(2 \epsilon)^{2 p+1}+\beta(2 \epsilon)^{2 p+1}\right\}, \\
& =\epsilon+C A 2(2 \epsilon)^{2 p+1}(1+\beta) .
\end{aligned}
$$

Therefore,

$$
\begin{aligned}
2 \epsilon & \leq \epsilon+2^{2 p+1} C A \epsilon^{2 p+1}(1+\beta), \\
1 & \leq 2^{2 p+1} C A \epsilon^{2 p}(1+2 \beta),
\end{aligned}
$$

which contradicts (2.20).

We now show that

$$
\|(u, v)\|_{\mathbb{L}^{a}\left(\left(0, T_{\max }\right), L^{r}\right)} \leq 2\left\{\|S(.)(\varphi, \psi)\|_{\mathbb{L}^{a}\left(\left(0, T_{0}\right), L^{r}\right)} .\right.
$$

If possible, assume that

$$
\|(u, v)\|_{\mathbb{L}^{a}\left(\left(0, T_{\max }\right), L^{r}\right)}>2\left\{\|S(\cdot)(\varphi, \psi)\|_{\mathbb{L}^{a}\left(\left(0, T_{0}\right), L^{r}\right)} .\right.
$$


From (2.19) and the argument of continuity, we have

$\|(u, v)\|_{\mathbb{L}^{a}\left((0, T), L^{r}\right)} \leq\|S(\cdot)(\varphi, \psi)\|_{\mathbb{L}^{a}\left((0, T), L^{r}\right)}+\left\{\left\|G_{h, u, v}\right\|_{L_{T}^{a} L_{x}^{r}}+\left\|G_{z, v, u}\right\|_{L_{T}^{a} L_{x}^{r}}\right\}$.

The estimates (2.26) and (2.27), imply that

$$
\|(u, v)\|_{\mathbb{L}^{a}\left(\left(0, T_{\max }\right), L^{r}\right)} \leq 2\left\{\left\|G_{h, u, v}\right\|_{L_{T}^{a} L_{x}^{r}}+\left\|G_{z, v, u}\right\|_{L_{T}^{a} L_{x}^{r}}\right\} .
$$

Now, the above inequality yields,

$$
X_{T_{\max }} \leq 2\left\{\|u\|_{L_{T_{\max }}^{a} L_{x}^{r}}^{2 p}+\beta\|v\|_{L_{T_{\max }}^{a} L_{x}^{r}}^{p}\|u\|_{L_{T_{\max }}^{a} L_{x}^{r}}^{p}\right\} G_{h, z, T},
$$

where $G_{h, z, T}=\left\|G_{h, u, v}\right\|_{L_{T}^{a} L_{x}^{r}}+\left\|G_{z, v, u}\right\|_{L_{T}^{a} L_{x}^{r}}$.

Finally, the estimates (2.22) and (2.29) give,

$$
C A X_{T_{\max }} \leq 2 C A\left((2 \epsilon)^{2 p}+\beta(2 \epsilon)^{2 p}\right) G_{h, z, T}=(1+\beta) 2^{2 p+1} C A \epsilon^{2 p} G_{h, z, T}<G_{h, z, T},
$$

which is a contradiction.

Note that, applying Holder's inequality, we have

$$
\left\|\left.|| u(t)\right|^{2 p} u(t)\right\|_{W^{1, r^{\prime}}} \leq C_{p}\|u(t)\|_{L^{r}}^{2 p}\|u(t)\|_{W^{1, r}} .
$$

Moreover,

$$
\left\|\left.|| u\right|^{p-1}|v|^{p+1} u\right\|_{W^{1, r^{\prime}}} \leq C_{p}^{\prime}\left\{\|u\|_{L^{r}}^{p}\|v\|_{L^{r}}^{p}+\|u\|_{L^{r}}^{p-1}\|v\|_{L^{r}}^{p+1}\right\}\left(\|u\|_{W^{1, r}}+\|v\|_{W^{1, r}}\right),
$$

where $r^{\prime}$ is the conjugate of $r$ and that $\nabla\left(|u|^{p+1}\right)=\left(\frac{p+1}{2}\right)|u|^{p-1}(\bar{u} \nabla u+u \overline{\nabla u})$.

We observe that

$$
\|\| u\left\|_{L^{r}}^{2 p}\right\| u\left\|_{W^{1, r}}\right\|_{L_{x}^{q^{\prime}}} \leq\|u\|_{L_{T}^{a} L_{x}^{r}}^{2 p}\|u\|_{L_{T}^{q} W^{1, r}}
$$

and

$$
\int_{0}^{T}\|u\|_{L_{x}^{r}}^{(p-1) q^{\prime}}\|v\|_{L_{x}^{r}}^{(p+1) q^{\prime}}\|u\|_{W^{1, r}}^{q^{\prime}} d t \leq\|u\|_{L_{T}^{a} L^{r}}^{(p-1) q^{\prime}}\|v\|_{L_{T}^{a} L^{r}}^{(p+1) q^{\prime}}\|u\|_{L_{T}^{q} W^{1, r}}^{q^{\prime}}
$$

where $q^{\prime}$ is the conjugate of $q$. Thus,

$\|h F(u, v)\|_{L^{q^{\prime}}\left((0, T), W^{1, r^{\prime}}\right)} \leq B_{p}\left(\|u\|_{L_{T}^{a} L_{x}^{r}}^{2 p}+\beta\|u\|_{L_{T}^{a} L^{r}}^{(p-1) q^{\prime}}\|v\|_{L_{T}^{a} L^{r}}^{(p+1) q^{\prime}}\right)\left\{\|u\|_{L_{T}^{q} W^{1, r}}+\|v\| \|_{L_{T}^{q} W^{1, r}}\right\}$.

Consequently

$$
\|(u, v)\|_{\mathbb{L}^{q}\left((0, T), W^{1, r}\right)}+\|(u, v)\|_{\mathbb{L}^{\infty}\left((0, T), H^{1}\right)} \leq \Lambda\|\|(\varphi, \psi) \|_{1,2} \quad \forall 0<T<T_{\max } .
$$

Moreover by continuity

$$
\|(u, v)\|_{\mathbb{L}^{q}\left(\left(0, T_{\max }\right), W^{1, r}\right)} \leq \Lambda\|\mid(\varphi, \psi)\| \|_{1,2},
$$


in particular, $(u, v) \in \mathbb{L}^{\infty}\left(\left(0, T_{\max }\right), H^{1}\right)$ so that $T_{\max }=\infty$ by the blow-up alternative.

Corollary 2.1. Assume (1.2) and $p>2 / n$ and $a, q, r$ be as defined in (2.7). Suppose that $h, z \in L^{\infty}(\mathbb{R})$ be such that $\|h\|_{L^{\infty}}+\|z\|_{L^{\infty}} \leq A$ for some $A>0$. Also let $\epsilon=\epsilon(A)$ and $\Lambda$ be as in Proposition 2.2. For given $(\phi, \psi) \in H^{1}\left(\mathbb{R}^{n}\right) \times H^{1}\left(\mathbb{R}^{n}\right)$, let $(u, v)$ be the corresponding solution of (2.3) defined on the maximal interval of existence $\left[0, T_{\max }\right)$. If there is $0<T<T_{\max }$ such that $\left\|\left(e^{i t \Delta} u(T), e^{i t \Delta} v(T)\right)\right\|_{\mathbb{L}^{a}\left((0, \infty) ; L^{r}\right)} \leq \epsilon$, then the solution $(u, v)$ is global, i.e. $T_{\max }=\infty$. Moreover

$$
\|(u, v)\|_{\mathbb{L}^{a}\left((T, \infty), L^{r}\right)} \leq 2 \epsilon, \quad\|(u, v)\|_{\mathbb{L}^{q}\left((T, \infty), W^{1, r}\right)} \leq \Lambda\|\mid(u(T), v(T))\| \|_{1,2} .
$$

Proof. If we apply Proposition 2.2 with $(\varphi, \psi)$ replaced by $(u(T), v(T))$ and $h(t), z(t)$ replaced by $h(t+T)$ and $z(t+T)$, it can be inferred that the solution $\left(w_{1}, w_{2}\right)$ of the system

$$
\left\{\begin{array}{l}
w_{1}(t)=e^{i t \Delta} u(T)+i \int_{0}^{t} e^{i(t-s) \Delta} h(s+T) F\left(w_{1}, w_{2}\right)(s) d s, \\
w_{2}(t)=e^{i t \Delta} v(T)+i \int_{0}^{t} e^{i(t-s) \Delta} z(s+T) F\left(w_{2}, w_{1}\right)(s) d s
\end{array}\right.
$$

is global and satisfies

$$
\left\|\left(w_{1}, w_{2}\right)\right\|_{\mathbb{L}^{a}\left((0, \infty) ; L^{r}\right)} \leq 2 \epsilon, \quad\left\|\left(w_{1}, w_{2}\right)\right\|_{\mathbb{L}^{q}\left((0, \infty) ; W^{1, r}\right)} \leq \Lambda\|\mid(u(T), v(T))\| \|_{1,2} .
$$

Now, if we define

$$
\begin{aligned}
& \tilde{u}= \begin{cases}u(t), & 0 \leq t \leq T, \\
w_{1}(t-T), & T<t<\infty,\end{cases} \\
& \tilde{v}= \begin{cases}v(t), & 0 \leq t \leq T, \\
w_{2}(t-T), & T<t<\infty,\end{cases}
\end{aligned}
$$

then it can be seen that $(\tilde{u}, \tilde{v})$ solves $(2.3)$ in $[0, \infty)$, thereby completing the proof.

In what follows we prove some more estimates that will be used in sequel.

Proposition 2.3. Assume $h \in C(\mathbb{R}, \mathbb{R})$ is a periodic function with period $\tau>0$ whose average is given by (1.9). Set $(q, r)$ be an admissible pair. Given $f \in L^{q^{\prime}}\left(\mathbb{R}, L^{r^{\prime}}\left(\mathbb{R}^{n}\right)\right)$, it follows that for every admissible pair $(\gamma, \rho)$

$$
\int_{0}^{t} h\left(\omega\left(s+t_{0}\right)\right) S(t-s) f(s) d s \quad \rightarrow \quad I(h) \int_{0}^{t} S(t-s) f(s) d s
$$

in $L^{\gamma}\left(\mathbb{R}, L^{\rho}\left(\mathbb{R}^{n}\right)\right)$, uniformly in $t_{0} \in \mathbb{R}$. 
Proof. A detailed proof of this result has been presented in [5]. For the sake of clarity, we just give a sketch here. Using the Strichartz estimate (2.2), we have that

$$
\| \int_{0}^{t} h\left(\omega\left(s+t_{0}\right) S(t-s) f(s) d s\left\|_{L^{\gamma}\left(\mathbb{R} ; L^{\rho}\left(\mathbb{R}^{n}\right)\right)} \leq C\right\| h\left\|_{L^{\infty}}\right\| f \|_{L^{q^{\prime}}\left(\mathbb{R} ; L^{r^{\prime}}\left(\mathbb{R}^{n}\right)\right)} .\right.
$$

So, by the density argument, it is enough to prove $(2.41)$ for $f \in C_{c}^{1}\left(\mathbb{R} ; \mathcal{S}\left(\mathbb{R}^{N}\right)\right)$. Defining, $\lambda(t):=h(t)-I(h)$ and $\Lambda(t)=\int_{0}^{t} \lambda(s) d s$, one has

$$
\frac{d}{d s} \Lambda\left(\omega\left(s+t_{0}\right)\right)=\omega \lambda\left(\omega\left(s+t_{0}\right)\right) .
$$

Now, integrating by parts and using the Strichartz estimate (2.2), it is easy to obtain

$$
\begin{aligned}
& \left\|\int_{0}^{t} \lambda\left(\omega\left(s+t_{0}\right)\right) S(t-s) f(s) d s\right\|_{L^{\gamma}\left(\mathbb{R} ; L^{\rho}\left(\mathbb{R}^{n}\right)\right)} \leq \\
& \frac{C}{|\omega|}\|\Lambda\|_{L^{\infty}}\left[\|f\|_{L^{\gamma}\left(\mathbb{R} ; L^{\rho}\left(\mathbb{R}^{n}\right)\right)}+\|f(0)\|_{L^{2}}+\left\|f_{t}-i \Delta f\right\|_{\left.L^{q^{\prime}\left(\mathbb{R} ; L^{r^{\prime}}\left(\mathbb{R}^{N}\right)\right)}\right]}\right] .
\end{aligned}
$$

Taking $|\omega| \rightarrow \infty$, the result of the proposition follows.

\section{Proof of the main Lemma}

The following Lemma plays a crucial role in the proof of the main result.

Lemma 3.1. Assume (1.2). Set the initial data $\varphi, \psi \in H^{1}\left(\mathbb{R}^{n}\right)$ and $t_{0}, \omega \in \mathbb{R}$, denote by $\left(u_{t_{0}, \omega}, v_{t_{0}, \omega}\right)$ the maximal solution of (2.3). Suppose $(U, V)$ be the maximal solution of (1.11) defined in $\left[0, S_{\max }\right)$. Let $0<T<S_{\max }$ and assume that $\left(u_{t_{0}, \omega}, v_{t_{0}, \omega}\right)$ exists on $[0, T]$ for $|\omega|$ sufficiently large and that

$$
\limsup _{|\omega| \rightarrow \infty} \sup _{t_{0} \in \mathbb{R}}\left\|\left(u_{t_{0}, \omega}, v_{t_{0}, \omega}\right)\right\|_{\mathbb{L}^{\infty}\left((0, T), H^{1}\right)}<\infty
$$

It follows that

$$
\sup _{t_{0} \in \mathbb{R}}\left\|u_{t_{0}, \omega}-U\right\|_{\mathbb{L}^{\gamma}\left((0, T), W^{1, \rho}\right)} \rightarrow 0, \text { when }|\omega| \rightarrow \infty
$$

for all admissible pair $(\gamma, \rho)$. In particular, $\left(u_{t_{0}, \omega}, v_{t_{0}, \omega}\right) \rightarrow(U, V)$ in $\mathbb{L}^{\infty}\left((0, T), H^{1}\right)$.

Proof. Symmetry of the system allows us to work for a single component. The estimates for the other component will be similar. We consider $|\omega| \geq L$, where $L$ is chosen sufficiently large so that

$$
\sup _{|\omega| \geq L} \sup _{t_{0} \in \mathbb{R}}\left\|\left(u_{t_{0}, \omega}, v_{t_{0}, \omega}\right)\right\|_{\mathbb{L}^{\infty}\left((0, T), H^{1}\right)}<\infty
$$


Let $r=2(p+1)$ and $q=\frac{4(p+1)}{n p}$ so that $(q, r)$ is an admissible pair. As the initial data for $u_{t_{0}, \omega}$ and $U$ are the same, we have,

$$
\begin{aligned}
u_{t_{0}, \omega}-U= & i \int_{0}^{t} S(t-s)\left[h F\left(u_{t_{0}, \omega}, v_{t_{0}, \omega}\right)(s)-I(h) F(U, V)(s)\right] d s \\
= & i \int_{0}^{t} S(t-s)\left[h\left|u_{t_{0}, \omega}\right|^{2 p} u_{t_{0}, \omega}(s)-I(h)|U|^{2 p} U(s)\right] d s \\
& +i \beta \int_{0}^{t} S(t-s)\left[h\left|u_{t_{0}, \omega}\right|^{p-1}\left|v_{t_{0}, \omega}\right|^{p+1} u_{t_{0}, \omega}(s)-I(h)|U|^{p-1}|V|^{p+1} U(s)\right] d s \\
= & : A+B .
\end{aligned}
$$

The estimates for $A$ follow from [5]. In fact, from [5] we have

$$
\|A\|_{L^{q}\left((0, t) ; L^{r}\right)}+\|A\|_{L^{\gamma}\left((0, t) ; L^{\rho}\right)} \leq C_{\omega}+C\left\|u_{t_{0}, \omega}-U\right\|_{L^{q^{\prime}}\left((0, t) ; L^{r}\right)},
$$

for all $0<t \leq T$, where $C_{\omega} \rightarrow 0$ as $|\omega| \rightarrow \infty$.

We move to estimate $B$ by writing it as

$$
\begin{aligned}
B= & i \beta \int_{0}^{t} S(t-s) h\left[\left|u_{t_{0}, \omega}\right|^{p-1}\left|v_{t_{0}, \omega}\right|^{p+1} u_{t_{0}, \omega}(s)-|U|^{p-1}|V|^{p+1} U(s)\right] d s \\
& +i \beta \int_{0}^{t} S(t-s)[h-I(h)]|U|^{p-1}|V|^{p+1} U(s) d s \\
& :=B_{1}+B_{2} .
\end{aligned}
$$

We note that $|U|^{p-1}|V|^{p+1} U \in L^{q^{\prime}}\left((0, T) ; L^{r^{\prime}}\left(\mathbb{R}^{n}\right)\right)$, because for $r=2 p+2, r^{\prime}=\frac{r}{2 p+1}$, using Hölder's inequality, one has

$$
\begin{aligned}
\int_{\mathbb{R}^{n}}|U|^{p r^{\prime}}|V|^{(p+1) r^{\prime}} d x & =\int_{\mathbb{R}^{n}}|U|^{\frac{p r}{2 p+1}}|V|^{\frac{(p+1) r}{2 p+1}} d x \\
& \leq\left(\int_{\mathbb{R}^{n}}|U|^{r} d x\right)^{\frac{p}{2 p+1}}\left(\int_{\mathbb{R}^{n}}|V|^{r} d x\right)^{\frac{p+1}{2 p+1}} \\
& =\|U\|_{L^{r}}^{\frac{p r}{2 p+1}}\|V\|_{L^{r}}^{\frac{(p+1) r}{2 p+1}}
\end{aligned}
$$

From Sobolev embedding, we have

$$
\|U\|_{L^{r}} \leq C\|U\|_{H^{\frac{n}{2}-\frac{n}{r}}} \leq\|U\|_{H^{1}},
$$

whenever $p<\frac{2}{(n-2)^{+}}$. Hence, we get

$\left\||U|^{p-1}|V|^{p+1} U\right\|_{L^{q^{\prime}\left((0, T) ; L^{r^{\prime}}\right)}}^{q^{\prime}} \leq \int_{0}^{T}\|U\|_{L^{r}}^{\frac{p r q^{\prime}}{(2 p+1) r^{\prime}}}\|V\|_{L^{r}}^{\frac{(p+1) r q^{\prime}}{(2 p+1) r^{\prime}}} d t=\int_{0}^{T}\|U\|_{L^{r}}^{p q^{\prime}}\|V\|_{L^{r}}^{(p+1) q^{\prime}} d t<\infty$ 
as required. Therefore, from Proposition 2.3 we conclude that

$$
\sup _{t_{0} \in \mathbb{R}}\left\|B_{2}\right\|_{L^{q}\left((0, T) ; L^{r}\right)}+\left\|B_{2}\right\|_{L^{\gamma}\left((0, T) ; L^{\rho}\right)} \rightarrow 0, \quad \text { as }|\omega| \rightarrow \infty .
$$

To estimate $B_{1}$ we proceed as follows. For $p>0$, we have that

$$
\begin{aligned}
\left.|| u_{t_{0}, \omega}\right|^{p-1}\left|v_{t_{0}, \omega}\right|^{p+1} u_{t_{0}, \omega}-|U|^{p-1}\left|V_{t_{0}, \omega}\right|^{p+1} U \mid & \leq\left(\left|u_{t_{0}, \omega}\right|^{p}\left|v_{t_{0}, \omega}\right|^{p}+|U|^{p}|V|^{p}\right)|| v_{t_{0}, \omega}|-| V|| \\
& \leq\left(\left|u_{t_{0}, \omega}\right|^{p}\left|v_{t_{0}, \omega}\right|^{p}+|U|^{p}|V|^{p}\right)\left|v_{t_{0}, \omega}-V\right| .
\end{aligned}
$$

Using Strichartz estimate, one obtains

$$
\left\|B_{1}\right\|_{L^{\gamma}\left((0, t) ; L^{\rho}\right)} \leq C\left\|\left(\left|u_{t_{0}, \omega}\right|^{p}\left|v_{t_{0}, \omega}\right|^{p}+|U|^{p}|V|^{p}\right)\left|v_{t_{0}, \omega}-V\right|\right\|_{L^{q^{\prime}}\left((0, t) ; L^{r^{\prime}}\right)} .
$$

Using Hölder's inequality (see [12]), and the fact that $r=2 p+2$, we get

$$
\left\|\left(\left|u_{t_{0}, \omega}\right|^{p}\left|v_{t_{0}, \omega}\right|^{p}+|U|^{p}|V|^{p}\right)\left|v_{t_{0}, \omega}-V\right|\right\|_{L^{r^{\prime}}} \leq\left(\left\|u_{t_{0}, \omega}\right\|_{L^{r}}\left\|v_{t_{0}, \omega}\right\|_{L^{r}}+\|U\|_{L^{r}}\|V\|_{L^{r}}\right)\left\|v_{t_{0}, \omega}-V\right\|_{L^{r}} .
$$

Inserting (3.12) in (3.11) and using Hölder's inequality in time variable, yields

$$
\begin{aligned}
& \left\|B_{1}\right\|_{L^{\gamma}\left((0, t) ; L^{\rho}\right)} \\
& \quad \leq C\left(\left\|u_{t_{0}, \omega}\right\|_{L^{\infty}\left((0, t) ; L^{r}\right)}\left\|v_{t_{0}, \omega}\right\|_{L^{\infty}\left((0, t) ; L^{r}\right)}+\|U\|_{L^{\infty}\left((0, t) ; L^{r}\right)}\|V\|_{L^{\infty}\left((0, t) ; L^{r}\right)}\right)\left\|v_{t_{0}, \omega}-V\right\|_{L^{q^{\prime}\left((0, t) ; L^{r}\right)}} .
\end{aligned}
$$

The estimate (3.13) and Sobolev embedding $H^{1}\left(\mathbb{R}^{n}\right) \hookrightarrow L^{r}\left(\mathbb{R}^{n}\right)$ imply

$$
\left\|B_{1}\right\|_{L^{\gamma}\left((0, t) ; L^{\rho}\right)} \leq C\left\|v_{t_{0}, \omega}-V\right\|_{L^{q^{\prime}\left((0, t) ; L^{r}\right)}} .
$$

From (3.6), (3.9) and (3.14), one obtains that

$$
\|B\|_{L^{\gamma}\left((0, t) ; L^{\rho}\right)} \leq C_{\omega}+C\left\|v_{t_{0}, \omega}-V\right\|_{L^{q^{\prime}}\left((0, t) ; L^{r}\right)},
$$

for all $0<t \leq T$, where $C_{\omega} \rightarrow 0$ as $|\omega| \rightarrow \infty$.

With the similar procedure for the admissible pair $(q, r)$ we get estimates analogous to $(3.15)$, to have

$$
\|B\|_{L^{q}\left((0, t) ; L^{r}\right)}+\|B\|_{L^{\gamma}\left((0, t) ; L^{\rho}\right)} \leq C_{\omega}+C\left\|v_{t_{0}, \omega}-V\right\|_{L^{q^{\prime}\left((0, t) ; L^{r}\right)}},
$$

for all $0<t \leq T$, where $C_{\omega} \rightarrow 0$ as $|\omega| \rightarrow \infty$.

Now from (3.4), combining the estimates (3.5) and (3.16), we get $\left\|u_{t_{0}, \omega}-U\right\|_{L^{q}\left((0, t) ; L^{r}\right)}+\left\|u_{t_{0}, \omega}-U\right\|_{L^{\gamma}\left((0, t) ; L^{\rho}\right)} \leq C_{\omega}+C\left\|u_{t_{0}, \omega}-U\right\|_{L^{q^{\prime}\left((0, t) ; L^{r}\right)}}+C\left\|v_{t_{0}, \omega}-V\right\|_{L^{q^{\prime}}\left((0, t) ; L^{r}\right)}$. 
With the analogous argument we get the similar estimate for the second component too, i.e.,

$\left\|v_{t_{0}, \omega}-V\right\|_{L^{q}\left((0, t) ; L^{r}\right)}+\left\|v_{t_{0}, \omega}-V\right\|_{L^{\gamma}\left((0, t) ; L^{\rho}\right)} \leq C_{\omega}+C\left\|v_{t_{0}, \omega}-V\right\|_{L^{q^{\prime}}\left((0, t) ; L^{r}\right)}+C\left\|u_{t_{0}, \omega}-U\right\|_{L^{q^{\prime}}\left((0, t) ; L^{r}\right)}$,

From (3.17) and (3.18), we conclude that

$$
\begin{aligned}
\left\|\left(u_{t_{0}, \omega}, v_{t_{0}, \omega}\right)-(U, V)\right\|_{\mathbb{L}^{q}\left((0, t) ; L^{r}\right)}+ & \left\|\left(u_{t_{0}, \omega}, v_{t_{0}, \omega}\right)-(U, V)\right\|_{\mathbb{L}^{\gamma}\left((0, t) ; L^{\rho}\right)} \\
& \leq C_{\omega}+C\left\|\left(u_{t_{0}, \omega}, v_{t_{0}, \omega}\right)-(U, V)\right\|_{\mathbb{L}^{q^{\prime}}\left((0, t) ; L^{r}\right)},
\end{aligned}
$$

for all $0<t \leq T$, where $C_{\omega} \rightarrow 0$ as $|\omega| \rightarrow \infty$.

From (3.19), we have that

$$
\left\|\left(u_{t_{0}, \omega}, v_{t_{0}, \omega}\right)-(U, V)\right\|_{\mathbb{L}^{q}\left((0, t) ; L^{r}\right)} \leq C_{\omega}+C\left\|\left(u_{t_{0}, \omega}, v_{t_{0}, \omega}\right)-(U, V)\right\|_{\mathbb{L}^{q^{\prime}}\left((0, t) ; L^{r}\right)} .
$$

Since, $q>q^{\prime}$, we have

$$
\left\|\left(u_{t_{0}, \omega}, v_{t_{0}, \omega}\right)-(U, V)\right\|_{\mathbb{L}^{q}\left((0, t) ; L^{r}\right)} \leq C C_{\omega} \rightarrow 0, \quad|\omega| \rightarrow \infty .
$$

Therefore, from (3.21) and (3.19) one can conclude that

$$
\sup _{t_{0} \in \mathbb{R}}\left\|\left(u_{t_{0}, \omega}, v_{t_{0}, \omega}\right)-(U, V)\right\|_{\mathbb{L}^{\gamma}\left((0, t) ; L^{\rho}\right)} \rightarrow 0, \quad|\omega| \rightarrow \infty,
$$

for all $0<t \leq T$ and for all admissible pairs $(\gamma, \rho)$.

Next, we move to prove convergence in the space $\mathbb{L}^{\gamma}\left((0, T) ; W^{1, \rho}\right)$. In other words, we prove the following

$$
\|\nabla[(u, v)-(U, V)]\|_{\mathbb{L} \gamma\left((0, T) ; L^{\rho}\right)} \rightarrow 0, \quad|\omega| \rightarrow \infty .
$$

Note that

$\| \nabla\left[(u, v)-(U, V)\left\|_{\mathbb{L}^{\gamma}\left((0, T) ; L^{\rho}\right)}=\right\| \nabla(u-U)\left\|_{\mathbb{L}^{\gamma}\left((0, T) ; L^{\rho}\right)}+\right\| \nabla(v-V) \|_{\mathbb{L}^{\gamma}\left((0, T) ; L^{\rho}\right)}\right.$.

We have,

$$
\begin{aligned}
\nabla(u-U)= & i \nabla \int_{0}^{t} S(t-s)\left[h|u|^{2 p} u_{t_{0}, \omega}(s)-I(h)|U|^{2 p} U(s)\right] d s \\
& +i \beta \nabla \int_{0}^{t} S(t-s)\left[h|u|^{p-1}|v|^{p+1} u(s)-I(h)|U|^{p-1}|V|^{p+1} U(s)\right] d s \\
= & : I_{1}+I_{2} .
\end{aligned}
$$

With the same technique as in [5], we obtain

$$
\left\|I_{1}\right\|_{L^{\gamma}\left((0, T) ; L^{\rho}\right)} \rightarrow 0 \quad|\omega| \rightarrow \infty .
$$


To estimate $I_{2}$, let us define $g(u, v)=|u|^{p-1} u|v|^{p+1}$, so that

$$
\begin{aligned}
\nabla g(u, v) & =\left(\begin{array}{c}
\frac{p+1}{2}|u|^{p-1}|v|^{p+1} \\
\frac{p-1}{2}|u|^{p-3}|v|^{p-1} u^{2}
\end{array}\right)\left(\begin{array}{c}
\nabla u \\
\nabla \bar{u}
\end{array}\right)+\left(\begin{array}{c}
\frac{p+1}{2}|u|^{p-1}|v|^{p-1} u \bar{v} \\
\frac{p-1}{2}|u|^{p-1}|v|^{p+1} u v
\end{array}\right)\left(\begin{array}{c}
\nabla v \\
\nabla \bar{v}
\end{array}\right) \\
& =: g_{1}^{\prime}(u, v) \cdot D u+g_{2}^{\prime}(u, v) \cdot D v .
\end{aligned}
$$

Now, using (3.27), we get

$$
\begin{aligned}
I_{2}= & i \beta \int_{0}^{t} S(t-s)\left[h\left(g_{1}^{\prime}(u, v) \cdot D u+g_{2}^{\prime}(u, v) \cdot D v\right)-I(h)\left(g_{1}^{\prime}(U, V) \cdot D U+g_{2}^{\prime}(U, V) \cdot D V\right)\right] d s \\
= & i \beta \int_{0}^{t} h S(t-s)\left[g_{1}^{\prime}(u, v) \cdot(D u-D U)+g_{2}^{\prime}(u, v) \cdot(D v-D V)\right] d s \\
& +i \beta \int_{0}^{t} h S(t-s)\left[\left(g_{1}^{\prime}(u, v)-g_{1}^{\prime}(U, V)\right) \cdot D U+\left(g_{2}^{\prime}(u, v)-g_{2}^{\prime}(U, V)\right) \cdot D V\right] d s \\
& +i \beta \int_{0}^{t}(h-I(h)) S(t-s)\left[g_{1}^{\prime}(U, V) \cdot D U+g_{2}^{\prime}(U, V) \cdot D V\right] d s \\
= & : J_{1}+J_{2}+J_{3} .
\end{aligned}
$$

Now, using Strichartz inequality, for any admissible pair $(\gamma, \rho)$, one has

$$
\begin{aligned}
\left\|J_{1}\right\|_{L^{\gamma}\left((0, T) ; L^{\rho}\right)} & \leq C\left\{\left\|g_{1}^{\prime}(u, v) \cdot(D u-D U)\right\|_{L^{q^{\prime}\left((0, T) ; L^{r^{\prime}}\right)}}+\left\|g_{2}^{\prime}(u, v) \cdot(D v-D V)\right\|_{L^{q^{\prime}\left((0, T) ; L^{r^{\prime}}\right)}}\right\} \\
& \leq C\left\{\left\|\left|g_{1}^{\prime}(u, v)\|(D u-D U) \mid\|_{L^{q^{\prime}\left((0, T) ; L^{r^{\prime}}\right)}}+\|\| g_{2}^{\prime}(u, v)\|(D v-D V)\| \|_{\left.L^{q^{\prime}\left((0, T) ; L^{r^{\prime}}\right)}\right\}} .\right.\right.\right.
\end{aligned}
$$

Since

$$
\begin{aligned}
& \left|g_{1}^{\prime}(u, v)\right| \leq C_{p}|u|^{p-1}|v|^{p+1}, \quad\left|g_{2}^{\prime}(u, v)\right| \leq \hat{C}_{p}|u|^{p}|v|^{p} \\
& |D u-D U|=\left|\left(\begin{array}{c}
\nabla(u-U) \\
\nabla \overline{(u-U)}
\end{array}\right)\right| \leq 2|\nabla(u-U)|, \\
& |D v-D V|=\left|\left(\begin{array}{c}
\nabla(v-V) \\
\nabla \overline{(v-V)}
\end{array}\right)\right| \leq 2|\nabla(v-V)|,
\end{aligned}
$$

we obtain from (3.29) that

$$
\begin{aligned}
\left\|J_{1}\right\|_{L^{\gamma}\left((0, T) ; L^{\rho}\right)} \leq & C_{p}\left\||u|^{p-1}|v|^{p+1}|\nabla(u-U)|\right\|_{L^{q^{\prime}}\left((0, T) ; L^{r^{\prime}}\right)}+\hat{C}_{p}\left\||u|^{p}|v|^{p}|\nabla(v-V)|\right\|_{L^{q^{\prime}\left((0, T) ; L^{r^{\prime}}\right)}} \leq \\
\leq & C_{p}\left\||u|^{p-1}|v|^{p+1}\right\|_{L^{\infty}\left((0, T) ; L^{\frac{p+1}{p}}\right)}\|\nabla(u-U)\|_{L^{q^{\prime}}\left((0, T) ; L^{r}\right)} \\
& +\hat{C}_{p}\left\||u|^{p}|v|^{p}\right\|_{L^{\infty}\left((0, T) ; L^{\frac{p+1}{p}}\right)}\|\nabla(v-V)\|_{L^{q^{\prime}\left((0, T) ; L^{r}\right)}} .
\end{aligned}
$$


Using Hölders inequality, we have

$$
\begin{aligned}
& \left\||u|^{p-1}|v|^{p+1}\right\|_{L^{\infty}\left((0, T) ; L^{\frac{p+1}{p}}\right)} \leq C_{p}\|u\|_{L^{\infty}\left((0, T) ; L^{r}\right)}^{p-1}\|v\|_{L^{\infty}\left((0, T) ; L^{r}\right)}^{p+1} \\
& \left\||u|^{p}|v|^{p}\right\|_{L^{\infty}\left((0, T) ; L^{\frac{p+1}{p}}\right)} \leq C_{p}\|u\|_{L^{\infty}\left((0, T) ; L^{r}\right)}^{p}\|v\|_{L^{\infty}\left((0, T) ; L^{r}\right)}^{p}
\end{aligned}
$$

Now, using the Sobolev embedding $H^{1} \hookrightarrow L^{r}$, we obtain from (3.32) and (3.31)

$$
\left\|J_{1}\right\|_{L^{\gamma}\left((0, T) ; L^{\rho}\right)} \leq C_{p}\|(\nabla(u-U), \nabla(v-V))\|_{\mathbb{L}^{q^{\prime}}\left((0, T), L^{r}\right)} .
$$

With the similar procedure, also for the admissible pair $(q, r)$, we obtain

$$
\left\|J_{1}\right\|_{L^{q}\left((0, T) ; L^{r}\right)} \leq C_{p}\|(\nabla(u-U), \nabla(v-V))\|_{\mathbb{L}^{q^{\prime}}\left((0, T), L^{r}\right)} .
$$

To estimate $J_{2}$, we use Strichartz estimate for the admissible pairs $(\gamma, \rho)$ and $(q, r)$, and the fact that the solution $(U, V) \in L^{q} W^{1, r}$ to obtain

$$
\begin{aligned}
\left\|J_{2}\right\|_{L^{\gamma}\left((0, T) ; L^{\rho}\right)}+\left\|J_{2}\right\|_{L^{q}\left((0, T) ; L^{r}\right)} \leq & C\left\|\left(g_{1}^{\prime}(u, v)-g_{1}^{\prime}(U, V)\right) \cdot D U\right\|_{L^{q^{\prime}\left((0, T) ; L^{r^{\prime}}\right)}} \\
& +C\left\|\left(g_{2}^{\prime}(u, v)-g_{2}^{\prime}(U, V)\right) \cdot D V\right\|_{L^{q^{\prime}}\left((0, T) ; L^{r^{\prime}}\right)} \\
\leq & C\left\|\left(g_{1}^{\prime}(u, v)-g_{1}^{\prime}(U, V)\right)\right\|_{L^{\frac{q}{q-2}}\left((0, T) ; L^{\frac{r}{r-2}}\right)}\|\nabla U\|_{L^{q}\left((0, T), L^{r}\right)} \\
& +C\left\|\left(g_{2}^{\prime}(u, v)-g_{2}^{\prime}(U, V)\right)\right\|_{L^{\frac{q}{q-2}}\left((0, T) ; L^{\frac{r}{r-2}}\right)}\|\nabla V\|_{L^{q}\left((0, T), L^{r}\right)} \\
\leq & C\left\|\left(g_{1}^{\prime}(u, v)-g_{1}^{\prime}(U, V)\right)\right\|_{L^{\frac{q}{q-2}}\left((0, T) ; L^{\frac{r}{r-2}}\right)} \\
& +C\left\|\left(g_{2}^{\prime}(u, v)-g_{2}^{\prime}(U, V)\right)\right\|_{L^{\frac{q}{q-2}}\left((0, T) ; L^{\frac{r}{r-2}}\right)} .
\end{aligned}
$$

We have that as $|\omega| \rightarrow \infty,\|u-U\|_{L_{t}^{\infty} L_{x}^{2}} \rightarrow 0$ and $\|v-V\|_{L_{t}^{\infty} L_{x}^{2}} \rightarrow 0$. Using the interpolation relation $\|u-U\|_{H^{s}} \leq\|u-U\|_{L^{2}}^{1-s}\|u-U\|_{H^{1}}^{s}$, we can conclude that $u \rightarrow U$ and $v \rightarrow V$ in $C\left([0, T] ; H^{s}\left(\mathbb{R}^{n}\right)\right)$ as $|\omega| \rightarrow \infty$ for $0 \leq s<1$. If $s$ is sufficiently close to 1 such that $s>\frac{n}{2}-\frac{n}{r}$, then using the Sobolev embedding $H^{s}\left(\mathbb{R}^{n}\right) \hookrightarrow L^{r}\left(\mathbb{R}^{n}\right)$, we have that $u \rightarrow U$ and $v \rightarrow V$ in $C\left([0, T] ; L^{r}\left(\mathbb{R}^{n}\right)\right)$ as $|\omega| \rightarrow \infty$.

Note that $\left\|g_{1}^{\prime}(u, v)\right\|_{\mathbb{L}^{\frac{r}{r-2}}} \leq C_{p}\left\||u|^{p-1}|v|^{p+1}\right\|_{L^{\frac{r}{r-2}}} \leq C\|u\|_{L^{r}}^{p-1}\|v\|_{L^{r}}^{p+1}<\infty$, and similar holds for $g_{2}^{\prime}(u, v)$. Now, from dominated convergence theorem, we obtain that the mappings $(u, v) \rightarrow g_{1}^{\prime}(u, v)$ and $(u, v) \rightarrow g_{2}^{\prime}(u, v)$ are continuous from $\mathbb{L}^{r}\left(\mathbb{R}^{n}\right) \rightarrow \mathbb{L}^{\frac{r}{r-2}}\left(\mathbb{R}^{n}\right)$ and consequently,

$\sup _{t_{0} \in \mathbb{R}}\left[\left\|g_{1}^{\prime}(u, v)-g_{1}^{\prime}(U, V)\right\|_{\mathbb{L}^{\infty}\left((0, T) ; L^{\frac{r}{r-2}}\right)}+\left\|g_{2}^{\prime}(u, v)-g_{2}^{\prime}(U, V)\right\|_{\mathbb{L}^{\infty}\left((0, T) ; L^{\frac{r}{r-2}}\right)}\right] \rightarrow 0, \quad|\omega| \rightarrow \infty$ 
From (3.35) and (3.36) we conclude that

$$
\sup _{t_{o} \in \mathbb{R}}\left[\left\|J_{2}\right\|_{L^{\gamma}\left((0, T) ; L^{\rho}\right)}+\left\|J_{2}\right\|_{L^{q}\left((0, T) ; L^{r}\right)}\right]=: C_{\omega} \rightarrow 0, \quad|\omega| \rightarrow \infty .
$$

It is easy to see that $g_{1}^{\prime}(U, V) \cdot D U+g_{2}^{\prime}(U, V) \cdot D V \in L^{q^{\prime}}\left((0, T) ; L^{r^{\prime}}\left(\mathbb{R}^{n}\right)\right)$, so by Proposition 2.3, we have

$$
\sup _{t_{o} \in \mathbb{R}}\left[\left\|J_{3}\right\|_{L^{\gamma}\left((0, T) ; L^{\rho}\right)}+\left\|J_{3}\right\|_{L^{q}\left((0, T) ; L^{r}\right)}\right]=: C_{\omega} \rightarrow 0, \quad|\omega| \rightarrow \infty .
$$

Combining the estimates (3.33), (3.34), (3.37) and (3.38), we can conclude as in (3.22) that

$$
\sup _{t_{0} \in \mathbb{R}}\|\nabla(u, v)-\nabla(U, V)\|_{\mathbb{L}^{\gamma}\left((0, T) ; L^{\rho}\left(\mathbb{R}^{n}\right)\right.} \rightarrow 0, \quad|\omega| \rightarrow \infty,
$$

for all admissible pairs $(\gamma, \rho)$.

Hence the result of the lemma follows from (3.22) and (3.39).

\section{Proof of the main Results.}

Proof of Theorem 1.2. Let $T \in\left(0, S_{\max }\right), A=\max \left\{\left\|\theta_{1}\right\|_{\infty},\left\|\theta_{2}\right\|_{\infty}\right\}$,

$$
M=2 \sup _{0 \leq t \leq T}\left|\|(U(t), V(t)) \mid\|_{1,2}\right.
$$

and $\delta=\delta(A, M)$ be given by Proposition 2.1. It follows by this proposition that $\left(u_{t_{0}, \omega}(t), v_{t_{0}, \omega}(t)\right)$ exists on $[0, \delta]$ and satisfies

$$
\left\|\left(u_{t_{0}, \omega}, v_{t_{0}, \omega}\right)\right\|_{\mathbb{L}^{\infty}\left((0, \delta), H^{1}\right)} \leq C \mid\|(\varphi, \psi)\| \|_{1,2} .
$$

Now Lemma 3.1 implies that

$$
\sup _{t_{0} \in \mathbb{R}}\left\|\left(u_{t_{0}, \omega}, v_{t_{0}, \omega}\right)-(U, V)\right\|_{\mathbb{L}^{\gamma}\left((0, \delta), W^{1, \rho}\right)} \rightarrow 0, \quad \text { when } \quad|w| \rightarrow \infty,
$$

for all admissible pair $(\gamma, \rho)$ and in particular

$$
\sup _{t_{0} \in \mathbb{R}}\left\|\left(u_{t_{0}, \omega}, v_{t_{0}, \omega}\right)-(U, V)\right\|_{\mathbb{L}^{\gamma}\left((0, \delta), H^{1}\right)} \rightarrow 0, \quad \text { when } \quad|w| \rightarrow \infty .
$$

Combining (4.1) and (4.3) we obtain that for $|\omega|$ sufficiently large

$$
\sup _{t_{0} \in \mathbb{R}}\|\|\left(u_{t_{0}, \omega}(\delta), v_{t_{0}, \omega}(\delta)\right)\|\|_{1,2} \leq \sup _{t_{0} \in \mathbb{R}}\|\mid(U(\delta), V(\delta))\| \|_{1,2}+\frac{M}{2} \leq M .
$$

Applying again Proposition 2.1 translated by $\delta$ and using (4.2), we have that $u_{t_{0}, \omega}(t)$ exists on $[0,2 \delta]$ and that

$$
\limsup _{|\omega| \rightarrow \infty} \sup _{t_{0} \in \mathbb{R}}\left\|\left(u_{t_{0}, \omega}, v_{t_{0}, \omega}\right)\right\|_{\mathbb{L}\left((0,2 \delta), H^{1}\right)}<\infty
$$


If $2 \delta<T$, iterating this argument, we deduce that

$$
\limsup _{|\omega| \rightarrow \infty} \sup _{t_{0} \in \mathbb{R}}\left\|\left(u_{t_{0}, \omega}, v_{t_{0}, \omega}\right)\right\|_{\mathbb{L}\left((0, T), H^{1}\right)}<\infty .
$$

The result then follows from Lemma 3.1.

Proof of Theorem 1.3. Let $\epsilon \in(0, \epsilon(A))$, where $\epsilon(A)$ is as in Proposition 2.2. For sufficiently large $T$, from (1.13), one gets that

$$
\|(U, V)\|_{\mathbb{L}^{a}\left((T, \infty) ; L^{r}\left(\mathbb{R}^{n}\right)\right)} \leq \frac{\epsilon}{4} .
$$

Applying the Proposition 2.2 to the global solution

$$
(\tilde{U}(t), \tilde{V}(t))=(U(t+T), V(t+T)),
$$

the inequality (2.13) yields

$$
\begin{aligned}
& \|S(\cdot)(U(T), V(T))\|_{\mathbb{L}^{a}\left((0, \infty) ; L^{r}\right)}=\|S(\cdot)(\tilde{U}(0), \tilde{V}(0))\|_{\mathbb{L}^{a}\left((0, \infty) ; L^{r}\right)} \\
& \quad \leq 2\|(\tilde{U}, \tilde{V})\|_{\mathbb{L}^{a}\left((0, \infty) ; L^{r}\right)}=2\|(U, V)\|_{\mathbb{L}^{a}\left((T, \infty) ; L^{r}\right)} \leq \frac{\epsilon}{2}
\end{aligned}
$$

Now, in the light of this inequality, using Corollary 2.1, we get that

$$
\|(U, V)\|_{\mathbb{L}^{a}\left((T, \infty) ; L^{r}\right)} \leq \Lambda \mid\|(U(T), V(T))\| \|_{1,2} .
$$

From Theorem 1.2, we have that

$$
\sup _{t_{0} \in \mathbb{R}}\left\|\left(u_{t_{0}, \omega}, v_{t_{0}, \omega}\right)-(U, V)\right\|_{\mathbb{L}^{\gamma}\left((0, T) ; W^{1, \rho}\right)} \rightarrow 0, \quad|\omega| \rightarrow \infty,
$$

for all $T<\infty$ and all admissible pairs $(\gamma, \rho)$. So, in particular, we have

$$
\sup _{t_{0} \in \mathbb{R}}\|\|\left(u_{t_{0}, \omega}(T), v_{t_{0}, \omega}(T)\right)-(U, V) \|_{1,2} \rightarrow 0, \quad|\omega| \rightarrow \infty .
$$

Therefore, we have, using (4.7) that

$$
\begin{aligned}
& \left\|S(\cdot)\left(u_{t_{0}, \omega}(T), v_{t_{0}, \omega}(T)\right)\right\|_{\mathbb{L}^{a}\left((0, \infty) ; L^{r}\right)} \\
& \leq\left\|S(\cdot)\left(u_{t_{0}, \omega}(T), v_{t_{0}, \omega}(T)\right)-S(\cdot)(U(T), V(T))\right\|_{\mathbb{L}^{a}\left((0, \infty) ; L^{r}\right)} \\
& \quad+\|S(\cdot)(U(T), V(T))\|_{\mathbb{L}^{a}\left((0, \infty) ; L^{r}\right)} \\
& \leq\|\|\left(u_{t_{0}, \omega}(T), v_{t_{0}, \omega}(T)\right)-(U(T), V(T))\|\|_{1,2}+\frac{\epsilon}{2} \leq \epsilon,
\end{aligned}
$$

for sufficiently large $|\omega|$.

Hence, from Corollary 2.1, we conclude that the solution $\left(u_{t_{0}, \omega}, v_{t_{0}, \omega}\right)$ is global and satisfies

$$
\sup _{t_{0} \in \mathbb{R}}\left\|\left(u_{t_{0}, \omega}, v_{t_{0}, \omega}\right)\right\|_{\mathbb{L}^{a}\left((T, \infty) ; L^{r}\right)} \leq 2 \epsilon
$$


and

$$
\left\|\left(u_{t_{0}, \omega}, v_{t_{0}, \omega}\right)\right\|_{\mathbb{L}^{q}\left((T, \infty) ; W^{1, r}\right)}+\left\|\left(u_{t_{0}, \omega}, v_{t_{0}, \omega}\right)\right\|_{\mathbb{L}^{\infty}\left((T, \infty) ; H^{1}\right)} \leq \Lambda\left\|\mid\left(u_{t_{0}, \omega}(T), v_{t_{0}, \omega}(T)\right)\right\|_{\left.\right|_{1,2}}
$$

Also, in view of corollary 2.1 and (4.5), we have

$$
\|(U, V)\|_{\mathbb{L}^{a}\left((T, \infty) ; L^{r}\right)} \leq \epsilon
$$

and

$$
\|(U, V)\|_{\mathbb{L}^{q}\left((T, \infty) ; W^{1, r}\right)}+\|(U, V)\|_{\mathbb{L}^{\infty}\left((T, \infty) ; H^{1}\right)} \leq \Lambda\|\|(U(T), V(T)) \|_{1,2}
$$

Let $M_{0}=\sup _{0 \leq t \leq T}\|(U(T), V(T))\|_{1,2}$. From (4.8), (4.10) and (4.12), it is easy to see that there exists $L>0$ sufficiently large such that

$$
\sup _{|\omega| \geq L} \sup _{t_{0} \in \mathbb{R}} \sup _{t \geq 0}\left\|||\left(u_{t_{0}, \omega}(t), v_{t_{0}, \omega}(t)\right)\left|\left\|_{1,2}+\sup _{t \geq 0}\right\|\right|(U(t), V(t))||_{1,2} \leq M_{1}<\infty .\right.
$$

In what follows, we prove that, for all admissible pairs $(\gamma, \rho),\left(u_{t_{0}, \omega}, v_{t_{0}, \omega}\right) \rightarrow(U, V)$ in $\mathbb{L}^{\gamma}\left((0, \infty), W^{1, \rho}\right)$ uniformly in $t_{0} \in \mathbb{R}$.

Let $|\omega| \gg 1$ so that the solution $\left(u_{t_{0}, \omega}, v_{t_{0}, \omega}\right)$ exists globally and fix $T>0$ to be chosen later. Note that,

$$
\begin{aligned}
\left\|\left(u_{t_{0}, \omega}, v_{t_{0}, \omega}\right)-(U, V)\right\|_{\mathbb{L}^{\gamma}\left((0, \infty), W^{1, \rho}\right)} \leq & \left\|\left(u_{t_{0}, \omega}, v_{t_{0}, \omega}\right)-(U, V)\right\|_{\mathbb{L}^{\gamma}\left((0, T), W^{1, \rho}\right)} \\
& +\left\|\left(u_{t_{0}, \omega}, v_{t_{0}, \omega}\right)-(U, V)\right\|_{\mathbb{L}^{\gamma}\left((T, \infty), W^{1, \rho}\right)} .
\end{aligned}
$$

From Theorem 1.2, we have that the first term in the right hand side of (4.16) converges to zero as $|\omega| \rightarrow \infty$. So, the convergence we are looking for would follow, if we can prove that, for every $\varepsilon>0$, there exists $T>0$ such that for $|\omega|$ sufficiently large

$$
\left\|\left(u_{t_{0}, \omega}, v_{t_{0}, \omega}\right)-(U, V)\right\|_{\mathbb{L}^{\gamma}\left((T, \infty), W^{1, \rho}\right)} \leq \varepsilon
$$

holds true.

Our objective from here onwards is to prove (4.17). Looking at the symmetry of the model under consideration, the estimate (4.17) would follow if we prove it for a single component, i.e., if we prove that for every $\varepsilon>0$, there exists $T>0$ such that for $|\omega|$ sufficiently large, the following holds

$$
\left\|u_{t_{0}, \omega}-U\right\|_{L^{\gamma}\left((T, \infty), W^{1, \rho}\right)} \leq \varepsilon
$$


Using Duhamel's formula, for all $t>0$, we have

$$
\begin{aligned}
u_{t_{0}, \omega}(T+t)-U(T+t)= & S(t)\left(u_{t_{0}, \omega}(T)-U(T)\right) \\
& +i \int_{0}^{t} S(t-s) \theta_{1}\left(\omega\left(T+s+t_{0}\right)\right) F\left(u_{t_{0}, \omega}, v_{t_{0}, \omega}\right)(T+s) d s \\
& -i I\left(\theta_{1}\right) \int_{0}^{t} S(t-s) F(U, V)(T+s) d s \\
= & : Q_{1}(t)+Q_{2}(t)+Q_{3}(t) .
\end{aligned}
$$

Using the Strichartz estimate, we obtain

$$
\begin{gathered}
\left\|Q_{1}(t)\right\|_{L^{\gamma}\left((0, \infty), W^{1, \rho}\right)} \leq C A\left\|u_{t_{0}, \omega}(T)-U(T)\right\|_{H^{1}} \rightarrow 0, \quad|\omega| \rightarrow \infty \\
\left\|Q_{2}(t)\right\|_{L^{\gamma}\left((0, \infty), W^{1, \rho}\right)} \leq C A\left[\left\|\left|u_{t_{0}, \omega}\right|^{2 p} u_{t_{0}, \omega}\right\|_{L^{q^{\prime}}\left((T, \infty) ; W^{1, r^{\prime}}\right)}\right. \\
\left.+\beta\left\|\left|u_{t_{0}, \omega}\right|^{p-1}\left|v_{t_{0}, \omega}\right|^{p+1} u_{t_{0}, \omega}\right\|_{L^{q^{\prime}}\left((T, \infty) ; W^{1, r^{\prime}}\right)}\right] \\
+
\end{gathered}
$$

and

$$
\begin{aligned}
\left\|Q_{3}(t)\right\|_{L^{\gamma}\left((0, \infty), W^{1, \rho}\right)} \leq C A[ & \left\||U|^{2 p} U\right\|_{L^{q^{\prime}\left((T, \infty) ; W^{1, r^{\prime}}\right)}} \\
& \left.+\beta\left\||U|^{p-1}|V|^{p+1} U\right\|_{L^{q^{\prime}}\left((T, \infty) ; W^{1, r^{\prime}}\right)}\right] .
\end{aligned}
$$

We have that, proceeding as in the proof of (3.23) (see (3.31) and (3.32) in particular)

$$
\left\|\left|u_{t_{0}, \omega}\right|^{2 p} u_{t_{0}, \omega}\right\|_{W^{1, r^{\prime}}} \leq C\left\|u_{t_{0}, \omega}\right\|_{L^{r}}^{2 p}\left\|u_{t_{0}, \omega}\right\|_{W^{1, r}},
$$

and

$$
\left\|\left|u_{t_{0}, \omega}\right|^{p-1}\left|v_{t_{0}, \omega}\right|^{p+1} u_{t_{0}, \omega}\right\|_{W^{1, r^{\prime}}} \leq C\left\|u_{t_{0}, \omega}\right\|_{L^{r}}^{p-1}\left\|v_{t_{0}, \omega}\right\|_{L^{r}}^{p+1}\left\|u_{t_{0}, \omega}\right\|_{W^{1, r}} .
$$

Now, applying Hölder's inequality in time variable and the definition of $r$ and $a$ in (1.12), we obtain that

$$
\left\|Q_{2}\right\|_{L^{\gamma}\left((0, \infty), W^{1, \rho}\right)} \leq C A\left\|\left(u_{t_{0}, \omega}, v_{t_{0}, \omega}\right)\right\|_{\mathbb{L}^{a}\left((T, \infty) ; L^{r}\right)}^{2 p}\left\|\left(u_{t_{0}, \omega}, v_{t_{0}, \omega}\right)\right\|_{\mathbb{L}^{q}\left((T, \infty) ; W^{1, r}\right)} .
$$

Using (4.11), (4.14) and (4.15), we get

$$
\left\|Q_{2}\right\|_{L^{\gamma}\left((0, \infty), W^{1, \rho}\right)} \leq C A(2 \epsilon)^{2 p} \Lambda M_{1} .
$$

With the similar argument, one obtains

$$
\left\|Q_{3}\right\|_{L^{\gamma}\left((0, \infty), W^{1, \rho}\right)} \leq C A(2 \epsilon)^{2 p} \Lambda M_{0} .
$$


Now, given $\varepsilon>0$, we choose sufficiently small $\epsilon>0$ such that $C A(2 \epsilon)^{2 p} \Lambda\left(M_{1}+M_{0}\right)<$ $\varepsilon / 3$ and $|\omega| \gg 1$, so that (4.19), (4.20), (4.26) and (4.27) yield

$$
\begin{aligned}
&\left\|u_{t_{0}, \omega}(t)-U(t)\right\|_{L^{\gamma}\left((T, \infty) ; W^{1, \rho}\right)}=\left\|u_{t_{0}, \omega}(T+t)-U(T+t)\right\|_{L^{\gamma}\left((0, \infty) ; W^{1, \rho}\right)} \\
& \leq\left\|Q_{1}(t)\right\|_{L^{\gamma}\left((0, \infty) ; W^{1, \rho}\right)}+\left\|Q_{2}(t)\right\|_{L^{\gamma}\left((0, \infty) ; W^{1, \rho}\right)} \\
& \quad+\left\|Q_{3}(t)\right\|_{L^{\gamma}\left((0, \infty) ; W^{1, \rho}\right)} \\
&<\varepsilon,
\end{aligned}
$$

as required, and this completes the proof.

Acknowledgments. Part of this research was done while M. Panthee was visiting the Institute of Mathematics, Federal University of Rio de Janeiro, Brazil. He wishes to thank for the support received during his visit.

\section{REFERENCES}

[1] F. K. Abdullaev, J. G. Caputo, R. A. Kraenkel, B. A. Malomed, Controlling collapse in BoseEinstein condensates by temporal modulation of the scattering length, Phys. Rev. A 67, 012605 (2003).

[2] L. Bergé, Wave collapse in physics: principles and applications to light and plasma waves, Phys. Rep. 303, (1998) 259-370.

[3] J. Bourgain, Global solutions of nonlinear Schrödinger equations, (American Mathematical Society, Providence), RI, (1999).

[4] X. Carvajal, M. Panthee, M. Scialom, On the critical KdV equation with time-oscillating nonlinearity, Differential and Integral Equations 24, (2011) 541-567.

[5] T. Cazenave, M. Scialom, A Schrödinger equation with time-oscillating nonlinearity, Revista Matemática Complutense 23, (2010) 321-339.

[6] T. Cazenave, An introduction to nonlinear Schrödinger equations (Textos de Métodos Matemáticos), 2nd edn, vol 26, Rio de Janeiro: Universidade Federal do Rio de Janeiro, (1993).

[7] T. Cazenave and F. Weissler, The Cauchy problem for the critical nonlinear Schrödinger equations in $H^{s}$, Nonlinear Analysis TMA 14, (1990), 807-836.

[8] J. Chen, B. Guo, Blow-up profile to the solutions of two-coupled Schrödinger equation with harmonic potencial, J. Math. Phys. 50, (2009), 023505.

[9] L. Fanelli, E. Montefusco, On the blow-up threshold for weakly coupled nonlinear Schrödinger equations, J. Phys. A: Math. Theor. 40, (2007) 14139-14150.

[10] V. V. Konotop, P. Pacciani, Collapse of solutions of the nonlinear Schrödinger equation with a time dependent nonlinearity: application to the Bose-Einstein condensates, Phys. Rev. Lett. 94, (2005) 240-405.

[11] M. Lakshmanan, T. Kanna, R. Radhakrishnan, Shape-changing collisions of coupled bright solitons in birifrigent optical fibers, Rep. Math. Phys. 46, (2000) 143-156. 
[12] F. Linares, G. Ponce, Introduction to Nonlinear Dispersive Equations, Universitex, Springer, New York (2009).

[13] L. Ma, L. Zhao, Sharp thresholds of blow-up and global existence for the coupled nonlinear Schrödinger equations, J. Math. Phys. 49, (2008) 062103.

[14] L. Ma, X. Song, L. Zhao, On global rough solutions to a non-linear Schrödinger system, Glasgow Math. J. 51, (2009) 499-511.

[15] T. Ozawa, Remarks on proofs of conservation laws for nonlinear Schrödinger equations, Calc. Var. Partial Differential Equations 25, (2006) 403-408.

[16] D. C. Roberts, A. C. Newell, Finite-time collapse of $N$ classical fields described by coupled nonlinear Schrödinger equations, Phys. Rev. E 74, (2008) 047602.

[17] C. Sulem, P. L. Sulem, The nonlinear Schrödinger equation: Self-focusing and wave collapse, (New York: Springer), (1999).

[18] Y. Tsutsumi, $L^{2}$-solutions for nonlinear Schrödinger equations and nonlinear group, Funkcialaj Ekvacioj, 30, (1987) 115-125.

[19] L. Xiaoguang, W. Yonghong, L. Shaoyong, A sharp threshold of blow-up for coupled nonlinear Schrödinger equations, J. Phys. A: Math. Theor. 43, (2010) 1-11.

[20] V. E. Zakharov, Collapse of Langmuir waves, Sov. Phys.-JETP 35, (1972) 908-14.

Instituto de Matemática - UfrJ Av. Horácio Macedo, Centro de Tecnologia Cidade Universitária, Ilha do Fundão, CaiXa Postal 68530 21941-972 Rio de Janeiro, RJ, BRASIL

E-mail address: carvajal@im.ufrj.br

E-mail address: pgamboa@im.ufrj.br

Centro de Matemática, Universidade do Minho, 4710-057, Braga, Portugal, And, IMECC-UNICAMP, 10383-859, CAMPINAS, SP, BrAZIL.

E-mail address: mpanthee@math.uminho.pt 